\title{
COMPOSITIONS OF PROKARYOTE COMMUNITES AND THEIR RELATIONSHIP TO PHYSIOCHEMICAL FACTORS IN DECEMBER IN CHAOHU LAKE AND THREE URBAN RIVERS IN CHINA
}

\author{
WU, L. ${ }^{1 *}-$ SHU, F. ${ }^{2}-$ OU, Z. ${ }^{1}-$ CHEN, Q. ${ }^{1}-$ WANG, L. ${ }^{1}-$ WANG, H. ${ }^{1}-\mathrm{XU}, \mathrm{Z}^{1}{ }^{*}$ \\ ${ }^{1}$ School of Life Science, Hefei Normal University, Hefei 230601, China \\ (phone/fax: +86-551-6367-4150) \\ ${ }^{2}$ Key Laboratory of Wetland Ecology and Environment Conservation of Lake Nansi, College of \\ Life Sciences, Qufu Normal University, Qufu 273100, China \\ (phone: +86-537-4456-415; fax: +86-537-4456-415) \\ *Corresponding authors \\ e-mail:wuli090121@126.com(L.Wu),xuzhongdong@hftc.edu.cn (Z.Xu); phonelfax:+86- \\ 551-6367-4150 \\ (Received $18^{\text {th }}$ Jan 2019; accepted $28^{\text {th }}$ Feb 2019)
}

\begin{abstract}
The aim of this study was to determine the impacts of anthropogenic disturbances on compositions of prokaryote communites (CPCs) in Chaohu Lake and its three urban tributaries, in China. Prokaryotic diversity in Chaohu Lake and its tributaries was high, and prokaryotic communities showed lower richness in Nanfei River than in Zhegao River and Hangbu River, with waterbody nutrient levels negatively corresponding to prokaryotic diversity. Except for a few rare prokaryotes, or where classification was unclear, the prokaryotes were distributed into 13 phyla and 924 genera. The dominant prokaryotic phyla were Proteobacteria (31.51\%), Bacteroidetes (26.50\%), Cyanobacteria (19.05\%), and Actinobacteria (10.71\%). Redundancy analysis (RDA) results showed CPCs, as well as abundances of Proteobacteria, Actinobacteria, and Firmicutes to be primarily affected by the concentration of total phosphorus (TP). Furthermore, Cyanobacteria showed a significant correlation with the concentrations of total nitrogen $(\mathrm{TN})$, nitrate $\left(\mathrm{NO}_{3}\right)$ and ammonia nitrogen $\left(\mathrm{NH}_{4}\right)$. $\mathrm{pH}$ was correlated with Planctomycetes, while Thermotogae was generally associated with water temperature (WT). These results suggest that trophic status could play an important role in shaping CPCs in both freshwater lakes and rivers. Ultimately, urbanization was found to cause the deterioration of water quality within Chaohu Lake and its tributaries.
\end{abstract}

Keywords: prokaryotic community structure, diversity, high-throughput sequencing, redundancy analysis, freshwater

\section{Introduction}

With continued worldwide urban expansion and rapid economic and industrial development, discharge from anthropogenic activities (municipal, industrial, and agricultural) continues to expose freshwater systems to large quantities of anthropogenic pollutants. This pollution has resulted in a notable deterioration of freshwater quality (Martinuzzi et al., 2014; Jordaan and Bezuidenhout, 2016). In aquatic ecosystems, prokaryotes drive the transformation and cycling of most biologically active elements, so they play a critical role in the regeneration and mobilization of nutrients in freshwater food webs (Newton et al., 2011; Ren et al., 2013). Short generation times, high diversity, and quick reaction to (and recovery from) environmental change make prokaryotes ideal indicators of stress in freshwater systems. Anthropogenic disturbances on freshwater systems have major repercussion on the 
overall prokaryotic structure and function of these habitats (Jordaan and Bezuidenhout, 2016). To better manage and maintain the ecological environments of freshwater systems, we need to more fully understand how prokaryotic community diversity and distribution characteristics function in these environments. In recent years, cultureindependent methods were used to characterize prokaryotic diversity. This approach avoids the cultivation bias and yields detailed insight into the prokaryotic diversity of diverse ecosystems (Ni et al., 2017; Wu et al., 2017; Xiang et al., 2018). The use of high-throughput sequencing has become an increasingly popular technique for the analysis of prokaryotic communities in a variety of freshwater ecosystems, and has led to the increased detection of rare prokaryotic species (Lin et al., 2012; Bashenkhaeva et al., 2015; Dai et al., 2016; Wu et al., 2017).

Chaohu Lake, one of the five largest freshwater lakes in China, is a shallow and subtropical freshwater system. Chaohu Lake has three urban-river tributaries-Nanfei, Zhegao, and Hangbu Rivers-which drain into the lake (Wang et al., 2011; Fig. 1). Chaohu Lake plays an important role in commercial fishing, water supply, irrigation, navigation, tourism, and recreational activity throughout the region. However, in recently years, due to the increase in anthropogenic activity within the lake's watershed, Chaohu Lake has suffered from severe pollution and eutrophication (Zan et al., 2011). This has resulted in large blooms of blue-green algae during the summer months, which have caused the production of highly toxic microcystins, and the subsequent fouling (and temporary interruption) of the local drinking water supply. The water quality of Chaohu Lake's three tributaries has also been adversely affected by human activityincluding agricultural runoff, urban development, and the construction of informal settlements. The establishment of water management strategies requires previous knowledge of the ecological dynamics of target communities in the water body. To date, however, only two studies (Bao et al., 2008; Wei et al., 2008) have analyzed the phylogenetic composition of bacterioplankton communities in Chaohu Lake. This was accomplished by partially sequencing cloned 16S rRNA genes and PCR-DGGE (denaturing gradient gel electrophoresis) analysis. No further research has attempted to characterize the compositions of prokaryote communites (CPCs) in Chaohu Lake by way of high-throughput sequencing, and the information on the CPCs of the lake's three tributaries is virtually nonexistent. Ultimately, this has restricted the creation of an effective water management plan. Thus, it is important to collect data and conduct research on prokaryotic diversity within Chaohu Lake and its surrounding watershed. Moreover, much of our current understanding of local CPCs is primarily based on interlake or inter-river investigations, with less attention being given to the areas where rivers drain into the lake.

The objectives of this project were as follows: 1) characterize the CPCs in Chaohu Lake and its tributaries using high-throughput sequencing; 2) compare the CPCs among Chaohu Lake and its tributaries, and evaluate the importance of physicochemical parameters in the structuring of CPCs; 3) provide basic information for the restoration of Chaohu Lake and its tributaries.

\section{Materials and methods}

\section{Study area}

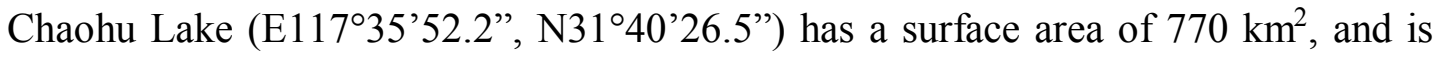
situated on the floodplain between the Yangtze River and the Huaihe River in the 
central Anhui Province of eastern China (Wang and Dou, 1998). The Nanfei River, Zhegao River, and Hangbu River, three tributaries of Chaohu Lake, flow through Hefei City, Zhegao Town, and Sanhe Town, respectively (Fig. 1). Farmland primarily surrounds the upper reaches of the three rivers, whereas cities and towns envelop the middle and lower reaches. Thus the middle and lower stretches are stressed from multiple anthropogenic sources, including untreated domestic and industrial wastewater discharge.

\section{Sampling sites and sample collection}

Samples were collected on 22 December 2017. We studied the Nanfei, Zhegao, and Hangbu Rivers, and established three sites along the upper reaches (U), middle reaches (M), and lower reaches (L) of all three rivers. Additionally, based on the shape and size of Chaohu Lake, three sites (I, II, III) were selected (Fig. 1). All stations were sampled within a 24-h period. The samples of three rivers were collected from the center of rivers. 500-ml water samples were collected at each sampling site for microbial DNA extraction. At each sampling site, 500-ml water samples were collected from surface waters (the top $50 \mathrm{~cm}$ ) and filtered through $0.2-\mu \mathrm{m}$-pore-size Isopore filters (Millipore, Billerica, MA, USA). All samples were collected only once. DNA extraction was carried out within $24 \mathrm{~h}$ from the time of sample collection. Simultaneously, $1 \mathrm{~L}$ water samples were collected at each sampling site for physicochemical characterization (Huang, 2000).

\section{Measurement of physical and chemical indices}

Water temperature was measured using a multi-parameter water quality monitoring sonde (YSI 6600, USA). One liter of water was collected to measure $\mathrm{pH}$, total phosphorus (TP), total nitrogen $(\mathrm{TN})$, nitrate $\left(\mathrm{NO}_{3}\right)$ and ammonia nitrogen $\left(\mathrm{NH}_{4}\right)$. All of these physiochemical factors were measured according to standard methods (Huang, 2000).

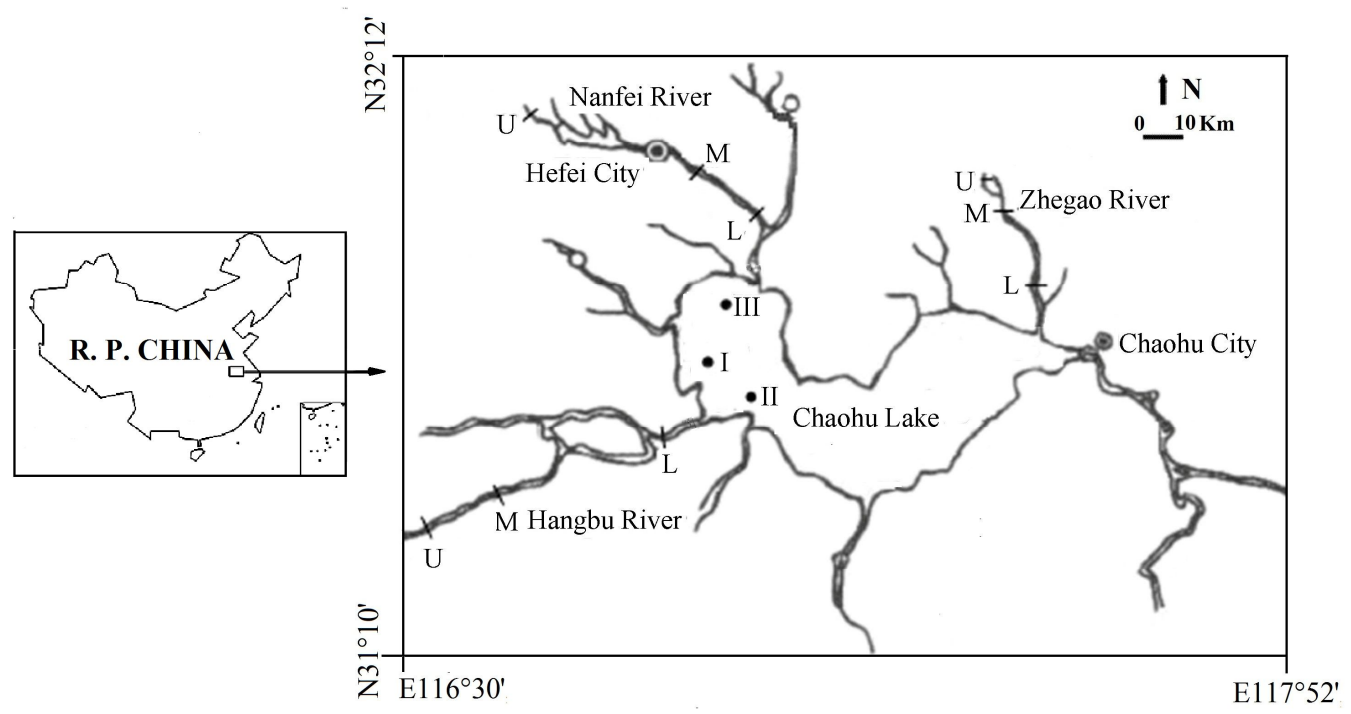

Figure 1. Sampling sites of Chaohu Lake and three rivers. $U$, upper reaches; $M$, middle reaches; and L, lower reaches; I, site I of Chaohu Lake; II, site II of Chaohu Lake; III, site III of Chaohu Lake 


\section{DNA extraction and high-throughput sequencing of prokaryotic $16 \mathrm{~S} r \mathrm{RNA}$ gene}

Genomic DNA of the water microbiota was extracted using the standard phenolchloroform method (Wu et al., 2017). The extracted DNA was diluted to $10 \mathrm{ng} / \mu \mathrm{L}$ and stored at $-40{ }^{\circ} \mathrm{C}$ for downstream use.

A prokaryotic universal primers $515 \mathrm{~F}$ and $909 \mathrm{R}$ with a $12 \mathrm{nt}$ unique barcode was used to amplify the V4-V5 hypervariable region of 16S rRNA gene for high-throughput sequencing according to a previous report (Ni et al., 2017). The PCR mixture $(25 \mu \mathrm{l})$ contained 1× PCR buffer, $1.5 \mathrm{mM} \mathrm{MgCl}$, deoxynucleoside triphosphate at $0.4 \mu \mathrm{M}$, primer at $1.0 \mu \mathrm{M}$ and $0.5 \mathrm{U}$ of Ex Taq (TaKaRa, China), and $10 \mathrm{ng}$ genomic DNA. The PCR amplification program included initial denaturation at $94{ }^{\circ} \mathrm{C}$ for $10 \mathrm{~min}$, followed by 30 cycles of $94^{\circ} \mathrm{C}$ for $40 \mathrm{~s}, 56^{\circ} \mathrm{C}$ for $60 \mathrm{~s}$, and $72{ }^{\circ} \mathrm{C}$ for $60 \mathrm{~s}$, and a final extension at $72{ }^{\circ} \mathrm{C}$ for $10 \mathrm{~min}$. Two PCR reactions were conducted for each sample and combined together after PCR amplification. PCR products were subjected to electrophoresis using $1 \%$ agarose gel. Each band showing the target size was excised and purified using a SanPrep DNA Gel Extraction Kit (Sangon Biotech, China) and quantified with Nanodrop. All samples were pooled together with equal molar amounts from each sample. The sequence samples were prepared using TruSeq DNA kit according to the manufacturer's instructions. The purified library was diluted, denatured, re-diluted, and mixed with PhiX (equal to $30 \%$ of the final DNA amount-as described in the Illumina library preparation protocols), and applied to an Illumina Miseq benchtop sequencer (Illumina, USA) at Guangdong Meilikang Bio-Science Ltd., China with the Reagent Kit (v2) $2 \times 250 \mathrm{bp}$ according to the manufacture manual.

\section{Data analysis}

The raw sequence data were merged using FLASH-1.2.8 software (Magoc and Salzberg, 2011). The merged sequences were trimmed and assigned to each sample based on their respective barcode sequences using QIIME Pipeline Version 1.7.0 (Caporaso et al., 2010). The sequences that are more than 300 bp of length, without ambiguous base ' $\mathrm{N}$ ', and more than 30 of the average base quality score were used for downstream analysis (Xiang et al., 2018). Sequences were clustered into operational taxonomic units (OTUs) at a $97 \%$ identity threshold. The aligned gene sequences were used for chimera check using the Uchime algorithm (Edgar et al., 2011). Taxonomy was assigned using the Ribosomal Database Project classifier (Wang et al., 2007). Shannon index was calculated using QIIME 1.7.0 based on the composition of OTUs. Principal coordinate analysis (PCoA) based on a weighted UniFrac distance matrix was conducted using QIIME 1.7.0. Redundancy analysis (RDA) with a Monte Carlo permutation test was carried out using the 'vegan' package in R (Oksanen et al., 2015). $P$-values $<0.05$ were considered statistically significant. We performed one-way analysis of variance (ANOVA) to test for significant differences in physicochemical characteristics. All statistical analyses were performed using SPSS 13.0.

\section{Results}

\section{Physicochemical analysis}

Concentrations of $\mathrm{TP}$ and $\mathrm{NH}_{4}$ exhibited maxima in the Nanfei River, while concentrations of $\mathrm{TN}$ and $\mathrm{NO}_{3}$ were greater in Chaohu Lake than in the three rivers (Table 1). According to the standard established by the Organisation for Economic and 
Co-operation and Development (OECD) (1982)-which is based on the concentration of total phosphorus (TP)-Chaohu Lake and its three associated rivers are eutrophic or hypereutrophic. The degree of eutrophication was as follows: Nanfei River $>$ Chaohu Lake $>$ Zhegao River $>$ Hangbu River.

Table 1. Physicochemical characteristics of Chaohu Lake and three rivers

\begin{tabular}{|c|c|c|c|c|c|c|c|}
\hline \multicolumn{2}{|c|}{$\begin{array}{c}\text { Physicochemical } \\
\text { variables }\end{array}$} & \multirow{2}{*}{$\frac{\mathbf{W T}\left({ }^{\circ} \mathbf{C}\right)}{3.50}$} & \multirow{2}{*}{$\begin{array}{l}\mathbf{p H} \\
7.92\end{array}$} & \multirow{2}{*}{$\begin{array}{c}\mathbf{T N}(\mathbf{m g} / \mathbf{L}) \\
1.36\end{array}$} & \multirow{2}{*}{$\begin{array}{c}\mathbf{N H}_{\mathbf{4}}(\mathbf{m g} / \mathbf{L}) \\
0.17\end{array}$} & \multirow{2}{*}{\begin{tabular}{|c|}
$\mathrm{NO}_{3}(\mathbf{m g} / \mathbf{L})$ \\
1.11
\end{tabular}} & \multirow{2}{*}{$\begin{array}{c}\mathbf{T P}(\mathbf{m g} / \mathbf{L}) \\
0.07\end{array}$} \\
\hline \multirow{4}{*}{$\begin{array}{l}\text { Hangbu } \\
\text { River }\end{array}$} & $\mathrm{HBU}$ & & & & & & \\
\hline & HBM & 4.50 & 7.54 & 0.82 & 0.04 & 0.31 & 0.10 \\
\hline & HBL & 4.90 & 7.59 & 1.17 & 0.13 & 0.34 & 0.08 \\
\hline & Mean \pm SD & $4.30 \pm 0.72$ & $7.68 \pm 0.21$ & $1.12 \pm 0.28$ & $0.11 \pm 0.06$ & $0.59 \pm 0.45$ & $0.08 \pm 0.02$ \\
\hline \multirow{4}{*}{$\begin{array}{l}\text { Zhegao } \\
\text { River }\end{array}$} & ZGU & 5.00 & 8.10 & 1.91 & 0.24 & 1.24 & 0.15 \\
\hline & ZGM & 4.80 & 8.01 & 0.86 & 0.06 & 0.61 & 0.06 \\
\hline & ZGL & 4.70 & 7.98 & 1.67 & 0.07 & 0.92 & 0.05 \\
\hline & Mean \pm SD & $4.83 \pm 0.15$ & $8.03 \pm 0.06$ & $1.48 \pm 0.55$ & $0.12 \pm 0.10$ & $0.92 \pm 0.32$ & $0.09 \pm 0.06$ \\
\hline \multirow{4}{*}{$\begin{array}{l}\text { Nanfei } \\
\text { River }\end{array}$} & & & & & & & 0.35 \\
\hline & NFM & & & & 0.00 & & 0.02 \\
\hline & NFL & 8.70 & 7.78 & 18.88 & 7.18 & 6.5 & 1.39 \\
\hline & Mean \pm SD & $6.57 \pm 3.52$ & $7.62 \pm 0.14$ & $10.22 \pm 8.95$ & $3.59 \pm 3.59$ & $3.90 \pm 3.06$ & $0.59 \pm 0.72$ \\
\hline \multirow{5}{*}{$\begin{array}{l}\text { Chaohu } \\
\text { Lake }\end{array}$} & $\mathrm{CH} \mathrm{I}$ & 4.00 & 7.96 & 7.96 & 0.40 & 4.17 & 0.19 \\
\hline & $\mathrm{CH}$ II & 4.50 & 7.99 & 4.17 & 1.29 & 1.37 & 0.23 \\
\hline & CH III & 4.40 & 7.75 & 28.44 & 8.80 & 8.58 & 0.23 \\
\hline & Mean $\pm \mathrm{SD}$ & $4.30 \pm 0.26$ & $7.90 \pm 0.13$ & $13.52 \pm 13.06$ & $3.49 \pm 4.61$ & $4.71 \pm 3.63$ & $0.21 \pm 0.02$ \\
\hline & $P$ & N.S. & 0.027 & N.S. & N.S. & N.S. & N.S. \\
\hline
\end{tabular}

$P$ : level of significance (ANOVA, $P<0.05$ ); N.S.: not significant

NFU: upper reaches of Nanfei River; NFM: middle reaches of Nanfei River; NFL: lower reaches of Nanfei River; ZGU: upper reaches of Zhegao River; ZGM: middle reaches of Zhegao River; ZGL: lower reaches of Zhegao River; HBU: upper reaches of Hangbu River; HBM: middle reaches of Hangbu River; HBL: lower reaches of Hangbu River; CH I: site I of Chaohu Lake; CH II: site II of Chaohu Lake; CH III: site III of Chaohu Lake

\section{Prokaryote community composition}

In total, 296,004 effective sequences and 39,350 OTUs (not include the averages number of OTUs in Chaohu Lake and three rivers) were obtained from these samples (Table 2). Chaohu Lake had the highest number of OTUs $(3,289)$ (Table 2). Additionally, prokaryote communities in Chaohu Lake showed higher richness than in the three rivers. The average Shannon diversity index for prokaryote communities in Chaohu Lake was 6.81. Among the three rivers, the highest observed prokaryotic diversity was in Zhegao River (7.60), followed by Hangbu River (6.99), and Nanfei River (6.21; Table 2). Thus, Chaohu Lake and its tributaries exhibited high prokaryotic diversity as well as an inverse relationship between water body trophic level and prokaryotic diversity.

Except for a small number of unclassified $(0.03 \%)$ and rare $(2.98 \%)$ prokaryotes, a total of 13 prokaryotic phyla were identified from Chaohu Lake and its tributaries. There were 13 dominant prokaryotic phyla, including Proteobacteria (31.51\%), Bacteroidetes (26.50\%), Cyanobacteria (19.05\%), Actinobacteria (10.71\%), 
Verrucomicrobia (2.73\%), Firmicutes (2.29\%), Planctomycetes (1.02\%), Chloroflexi $(0.82 \%)$, Acidobacteria $(0.62 \%)$, Chlorobi $(0.51 \%)$, Thermotogae $(0.50 \%)$, Crenarchaeota $(0.38 \%)$, and Euryarchaeota $(0.35 \%$; Fig. $2 A$ and Table 3). The Proteobacteria were distributed (in descending order) as Betaproteobacteria (19.88\%), Alphaproteobacteria (6.41\%), Gammaproteobacteria (4.47\%) and Deltaproteobacteria $(0.57 \%)$. Within the Bacteroidetes phylum, Flavobacteriia (17.75\%), Sphingobacteriia $(7.11 \%)$ and Bacteroidia $(1.16 \%)$ were the most frequently detected. Nostocophycideae $(8.70 \%)$, Synechococcophycideae (1.55\%), and Oscillatoriophycideae (1.24\%) were the dominant classes of Cyanobacteria phylum; Actinobacteria (9.73\%) and Verrucomicrobiae (1.57\%) were the main classes of the Actinobacteria and Verrucomicrobia phyla; the Firmicutes phylum predominantly consisted of the Clostridia (1.20\%) and Bacilli classes (1.00\%; Table 3).

Table 2. The richness and diversity of the prokaryotic community in Chaohu Lake and its tributaries

\begin{tabular}{c|c|c|c|c}
\hline & Site & Number of sequences & No. of OTU (97\% identity) & Shannon index \\
\hline \multirow{5}{*}{ Hangbu River } & HBU & 32615 & 2551 & 7.31 \\
& HBM & 16838 & 2010 & 6.9 \\
& HBL & 8918 & 1592 & 6.75 \\
& Average & 19457 & 2051 & 6.99 \\
\hline \multirow{5}{*}{ Zhegao River } & ZGU & 17535 & 2411 & 6.63 \\
& ZGM & 14868 & 2317 & 7.84 \\
& ZGL & 7465 & 1748 & 8.34 \\
& Average & 13289 & 2159 & 7.60 \\
\hline \multirow{5}{*}{ Nanfei River } & NFU & 24885 & 2685 & 6.75 \\
& NFM & 15424 & 2653 & 7.05 \\
& NFL & 7582 & 1678 & 4.82 \\
& Average & 15964 & 2339 & 6.21 \\
\hline \multirow{5}{*}{ Chaohu Lake } & CH I & 30711 & 3533 & 6.34 \\
& CH II & 28419 & 3720 & 7.77 \\
& CH III & 16743 & 2614 & 6.33 \\
& Average & 25291 & 3289 & 6.81 \\
\hline Total & & 296004 & 39350 & \\
\hline
\end{tabular}

Abbreviations are the same as those in Table 1

There was a distinct difference in the distribution of the dominant prokaryotic phyla in Chaohu Lake and its tributaries. For example, Proteobacteria exhibited the highest abundance in all of the collection stations-especially in Nanfei River-while Bacteroidetes abundance was highest in Hangbu River. Furthermore, Chaohu Lake and Zhegao River had high abundances of Cyanobacteria, Nanfei River had a high abundance of Firmicutes, and the middle reaches of Zhegao River (ZGM) had a high abundance of Verrucomicrobia. The upper reaches of Hangbu River (HBU) and upper reaches of Nanfei River (NFU) exhibited lower nutrient levels than the other stations, and also had the lowest abundance of Acidobacteria (Fig. 2A and Table 3). 
Table 3. Percentage of the most abundant phyla, classes, and genera in each sampling station (\%)

\begin{tabular}{|c|c|c|c|c|c|c|c|c|c|c|c|c|c|}
\hline Taxon & HBU & HBM & HBL & ZGU & ZGM & ZGL & NFU & NFM & NFL & CH I & CH II & CH III & $\begin{array}{l}\text { Percentage of } \\
\text { the total } \\
\text { prokaryotic } \\
\text { abundant }(\%)\end{array}$ \\
\hline Proteobacteria & 0.2304 & 0.3724 & 0.290 & 0.371 & 0.240 & 0.244 & 0.4905 & 0.5353 & 0.4877 & 0.154 & 0.1693 & 0.1959 & 31.5 \\
\hline Bacteroidetes & 0.6896 & 0.4351 & 0.491 & 0.170 & 0.169 & 0.110 & 0.3589 & 0.245 & 0.1195 & 0.097 & 0.1483 & 0.1463 & 26.5 \\
\hline Cyanobacteria & 0.0142 & 0.0625 & 0.020 & 0.222 & 0.197 & 0.309 & 0.052 & 0.0294 & 0.0667 & 0.525 & 0.4336 & 0.3536 & 19.1 \\
\hline Actinobacteria & 0.0098 & 0.0602 & 0.083 & 0.094 & 0.118 & 0.169 & 0.013 & 0.0439 & 0.1947 & 0.124 & 0.1611 & 0.2142 & 10.7 \\
\hline Verrucomicrobia & 0.0021 & 0.0066 & 0.035 & 0.028 & 0.137 & 0.038 & 0.0056 & 0.0052 & 0.0137 & 0.035 & 0.0161 & 0.0048 & 2.73 \\
\hline Firmicutes & 0.0171 & 0.0189 & 0.019 & 0.018 & 0.025 & 0.026 & 0.0296 & 0.0473 & 0.0235 & 0.013 & 0.0177 & 0.0201 & 2.29 \\
\hline Planctomycetes & 0.0010 & 0.0014 & 0.004 & 0.052 & 0.026 & 0.015 & 0.0014 & 0.0013 & 0.0083 & 0.006 & 0.0024 & 0.0041 & 1.02 \\
\hline Chloroflexi & 0.0030 & 0.003 & 0.005 & 0.007 & 0.005 & 0.019 & 0.0041 & 0.0054 & 0.0231 & 0.007 & 0.0068 & 0.0105 & 0.82 \\
\hline Acidobacteria & 0.0036 & 0.0042 & 0.007 & 0.005 & 0.007 & 0.008 & 0.0061 & 0.0106 & 0.0078 & 0.004 & 0.0048 & 0.0062 & 0.62 \\
\hline Chlorobi & 0.0011 & 0.0015 & 0.004 & 0.0008 & 0.020 & 0.005 & 0.0016 & 0.0016 & 0.0102 & 0.004 & 0.0042 & 0.0066 & 0.51 \\
\hline Thermotogae & 0.0048 & 0.0043 & 0.005 & 0.004 & 0.006 & 0.005 & 0.0047 & 0.0082 & 0.007 & 0.003 & 0.0043 & 0.0045 & 0.50 \\
\hline Crenarchaeota & 0.0036 & 0.0038 & 0.005 & 0.002 & 0.004 & 0.006 & 0.0051 & 0.0034 & 0.0044 & 0.002 & 0.0029 & 0.0034 & 0.38 \\
\hline Euryarchaeota & 0.0032 & 0.0031 & 0.004 & 0.001 & 0.004 & 0.003 & 0.0031 & 0.0056 & 0.0057 & 0.003 & 0.0027 & 0.0029 & 0.35 \\
\hline Taxon & HBU & HBM & HBL & ZGU & ZGM & ZGL & NFU & NFM & NFL & CH I & CH II & CH III & $\begin{array}{c}\text { Percentage of } \\
\text { the total } \\
\text { prokaryotic } \\
\text { abundant (\%) }\end{array}$ \\
\hline Proteobacteria;c__Betaproteobacteria & 0.1726 & 0.3235 & 0.219 & 0.140 & 0.143 & 0.123 & 0.3052 & 0.3384 & 0.3578 & 0.053 & 0.0863 & 0.1237 & 19.9 \\
\hline Proteobacteria;c__Gammaproteobacteria & 0.0453 & 0.0211 & 0.035 & 0.018 & 0.031 & 0.029 & 0.1422 & 0.1389 & 0.0305 & 0.011 & 0.0137 & 0.020 & 4.47 \\
\hline Proteobacteria;c__Alphaproteobacteria & 0.0087 & 0.0233 & 0.029 & 0.204 & 0.055 & 0.082 & 0.0339 & 0.0447 & 0.0922 & 0.085 & 0.0649 & 0.0456 & 6.41 \\
\hline Proteobacteria;c__Deltaproteobacteria & 0.0032 & 0.0036 & 0.006 & 0.007 & 0.007 & 0.008 & 0.0053 & 0.0104 & 0.0061 & 0.004 & 0.0038 & 0.0045 & 0.57 \\
\hline Bacteroidetes;c__Flavobacteriia & 0.6459 & 0.278 & 0.413 & 0.08 & 0.074 & 0.03 & 0.2989 & 0.1701 & 0.0343 & 0.022 & 0.0554 & 0.0275 & 17.8 \\
\hline
\end{tabular}




\begin{tabular}{|c|c|c|c|c|c|c|c|c|c|c|c|c|c|}
\hline Bacteroidetes;c__Sphingobacteriia & 0.0258 & 0.1424 & 0.059 & 0.076 & 0.08 & 0.063 & 0.0423 & 0.0541 & 0.0677 & 0.063 & 0.0784 & 0.1009 & 7.11 \\
\hline Bacteroidetes;c_Bacteroidia & 0.0137 & 0.011 & 0.015 & 0.008 & 0.01 & 0.011 & 0.014 & 0.0134 & 0.0115 & 0.007 & 0.0089 & 0.0143 & 1.16 \\
\hline Cyanobacteria;c_Nostocophycideae & 0.0035 & 0.0024 & 0.003 & 0.006 & 0.003 & 0.133 & 0.0035 & 0.0029 & 0.0051 & 0.345 & 0.2285 & 0.3072 & 8.70 \\
\hline Cyanobacteria;c_Oscillatoriophycideae & 0.0043 & 0.0029 & 0.003 & 0.010 & 0.009 & 0.006 & 0.0033 & 0.0033 & 0.0459 & 0.038 & 0.0185 & 0.0049 & 1.24 \\
\hline Cyanobacteria;c_Synechococcophycideae & 0.0009 & 0.0007 & 0.001 & 0.121 & 0.002 & 0.014 & 0.0008 & 0.001 & 0.0024 & 0.022 & 0.0126 & 0.0077 & 1.55 \\
\hline Actinobacteria;c_Actinobacteria & 0.0085 & 0.0538 & 0.068 & 0.078 & 0.104 & 0.147 & 0.0111 & 0.0412 & 0.1894 & 0.105 & 0.1566 & 0.2055 & 9.73 \\
\hline Firmicutes;c__Bacilli & 0.0062 & 0.0082 & 0.006 & 0.008 & 0.011 & 0.011 & 0.0154 & 0.0274 & 0.0086 & 0.005 & 0.0072 & 0.0073 & 1.01 \\
\hline Firmicutes;c_Clostridia & 0.0102 & 0.0104 & 0.013 & 0.009 & 0.013 & 0.014 & 0.0127 & 0.0191 & 0.0142 & 0.008 & 0.0091 & 0.012 & 1.2 \\
\hline Verrucomicrobia;c_Verrucomicrobiae & 0.0004 & 0.0018 & 0.025 & 0.013 & 0.119 & 0.013 & 0.0026 & 0.0029 & 0.0004 & 0.009 & 0.0006 & 0.0004 & 1.57 \\
\hline Verrucomicrobia;c_Opitutae & 0.0008 & 0.0035 & 0.007 & 0.009 & 0.010 & 0.015 & 0.0012 & 0.0006 & 0.012 & 0.015 & 0.0144 & 0.0026 & 0.76 \\
\hline Taxon & HBU & HBM & HBL & ZGU & ZGM & ZGL & NFU & NFM & NFL & CH I & CH II & CH III & $\begin{array}{l}\text { Percentage of } \\
\text { the total } \\
\text { prokaryotic } \\
\text { abundant (\%) }\end{array}$ \\
\hline Proteobacteria;g_Limnohabitans & 0.1043 & 0.0558 & 0.096 & 0.055 & 0.053 & 0.046 & 0.0897 & 0.0651 & 0.0092 & 0.008 & 0.0284 & 0.0586 & 5.57 \\
\hline Proteobacteria;g__Hydrogenophaga & 0.0066 & 0.2001 & 0.006 & 0.001 & 0.008 & 0.005 & 0.0929 & 0.0194 & 0.1916 & 0.0009 & 0.0019 & 0.0022 & 4.47 \\
\hline Proteobacteria;Other & 0.0373 & 0.035 & 0.04 & 0.023 & 0.046 & 0.036 & 0.0334 & 0.0689 & 0.0877 & 0.013 & 0.0226 & 0.036 & 3.98 \\
\hline Proteobacteria;g__ & 0.0013 & 0.0011 & 0.006 & 0.166 & 0.003 & 0.066 & 0.0021 & 0.0032 & 0.0731 & 0.069 & 0.0527 & 0.0306 & 3.94 \\
\hline Proteobacteria;g_Pseudomonas & 0.0204 & 0.0041 & 0.011 & 0.002 & 0.002 & 0.001 & 0.058 & 0.0171 & 0.0026 & 0.0006 & 0.001 & 0.0043 & 1.04 \\
\hline Bacteroidetes;g_Flavobacterium & 0.640 & 0.2638 & 0.407 & 0.069 & 0.051 & 0.022 & 0.2933 & 0.1656 & 0.0108 & 0.012 & 0.0403 & 0.018 & 16.6 \\
\hline Bacteroidetes;g__ & 0.0058 & 0.016 & 0.016 & 0.047 & 0.046 & 0.020 & 0.0129 & 0.0121 & 0.0222 & 0.014 & 0.0277 & 0.0687 & 2.57 \\
\hline Cyanobacteria;g_Dolichospermum & 0.0033 & 0.0024 & 0.003 & 0.006 & 0.003 & 0.132 & 0.0035 & 0.0029 & 0.0049 & 0.342 & 0.2258 & 0.3043 & 8.62 \\
\hline Cyanobacteria;g__ & 0.0032 & 0.0497 & 0.008 & 0.055 & 0.169 & 0.092 & 0.0393 & 0.003 & 0.0104 & 0.077 & 0.1224 & 0.0223 & 5.43 \\
\hline Cyanobacteria;g_Prochlorococcus & 0.0009 & 0.0006 & 0.001 & 0.117 & 0.002 & 0.013 & 0.0008 & 0.0008 & 0.0022 & 0.021 & 0.012 & 0.0073 & 1.49 \\
\hline Cyanobacteria;g__ & 0.0009 & 0.0035 & 0.003 & 0.013 & 0.006 & 0.039 & 0.0026 & 0.0077 & 0.0017 & 0.024 & 0.0358 & 0.0042 & 1.17 \\
\hline Actinobacteria;g & 0.0055 & 0.0465 & 0.056 & 0.069 & 0.09 & 0.132 & 0.0078 & 0.0237 & 0.168 & 0.096 & 0.1416 & 0.1900 & 8.56 \\
\hline
\end{tabular}


We identified 924 genera in 12 samples, with 12 dominant genera including Flavobacterium (16.60\%), Dolichospermum (8.62\%), Limnohabitans (5.57\%), Hydrogenophaga (4.47\%), Prochlorococcus (1.49\%), Pseudomonas (1.04\%), and 6 unidentified genera. These 6 identified genera included 3 dominant genera of Proteobacteria, 2 dominant genera of Cyanobacteria, 1 dominant genus of Bacteroidetes (Table 3). The changes in CPCs were mainly concentrated in Proteobacteria, Bacteroidetes, and Cyanobacteria.

A

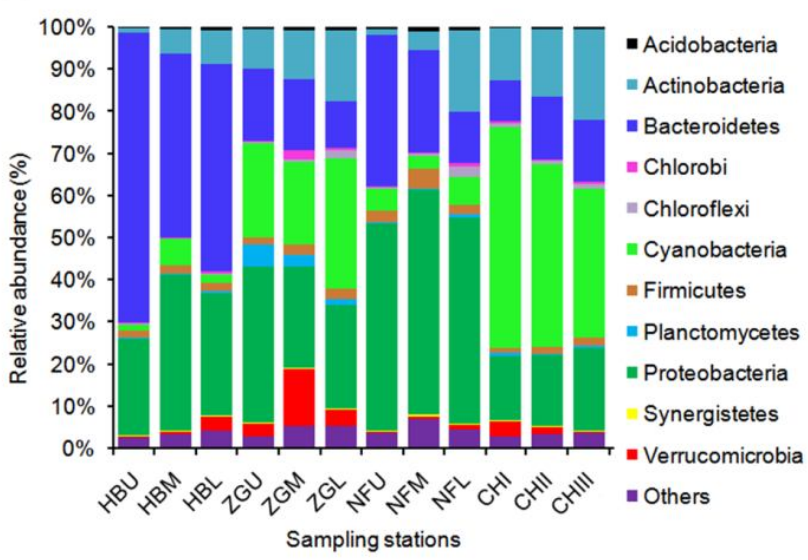

B

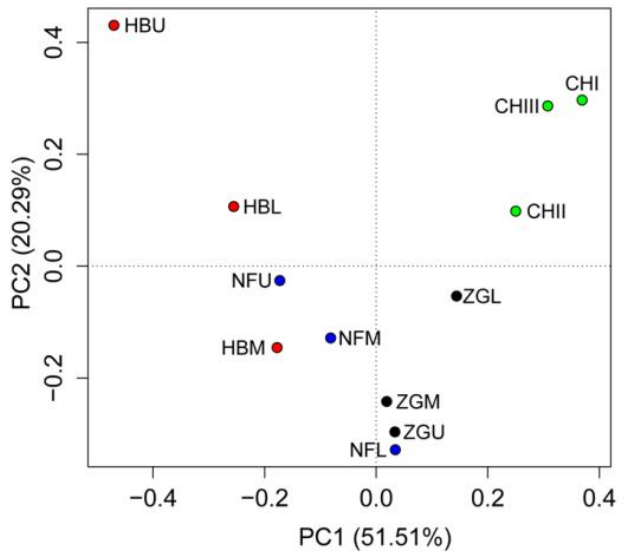

Figure 2. Relative abundances of dominant phyla $(A)$ and principal coordinate analysis $(P C o A)$ profile (B) of samples. The PCoA profile was drawn based on a weighted UniFrac distance matrix. Abbreviations are the same as those in Table 1

The results of PCoA showed a strong clustering of samples from Chaohu Lake, and a dispersed distribution among the rivers samples. This likely indicates a higher susceptibility among prokaryotic communities in Chaohu Lake than in the three rivers (Fig. 2B). Furthermore, the CPCs in Chaohu Lake were different from those in the three rivers and the spatial change patterns for prokaryotic communities were lake-specific and river-specific. This could imply that CPCs exhibit a high degree of spatial heterogeneity between the lake and the rivers.

\section{Relationship between prokaryotic communities and environmental factors}

According to the RDA analyses, $66 \%$ of the cumulative variance in the speciesenvironment relationship was explained by the first two RDA axes. Furthermore, following forward selection and the Monte Carlo test, the prokaryotic community showed a significant correlation with the concentration of TP $(p<0.01$; Fig. 3 and Table 4). For example, among the 13 phyla, Proteobacteria, Actinobacteria, and Firmicutes were primarily affected by the concentration of total phosphorus (TP; $p<0.05, p<0.05, p<0.05$, respectively). In contrast, Cyanobacteria showed a significant correlation with $\mathrm{TN}(p<0.01), \mathrm{NH}_{4}(p<0.01)$, and $\mathrm{NO}_{3}(p<0.05)$. Planctomycete showed a correlation with $\mathrm{pH}(p<0.05)$, and Thermotogae was generally associated to WT ( $p<0.05 ;$ Fig. 3 and Table 4). Bacteroidetes, Acidobacteria, Chlorobi, Verrucomicrobia, Euryarchaeota, Chloroflexi, and Crenarchaeota exhibited no correlation with the examined physicochemical factors. 
Table 4. Correlation matrix showing the relationship between prokaryotic community / 6 prokaryotic phyla and significantly associated environmental factors

\begin{tabular}{c|c|c|c}
\hline & & Axis 1 & Axis 2 \\
\hline Prokaryotic community & $\mathrm{TP}(\mathrm{mg} / \mathrm{L})$ & -0.59 & -0.80 \\
\hline Proteobacteria & $\mathrm{TP}(\mathrm{mg} / \mathrm{L})$ & 0.67 & 0.64 \\
Cyanobacteria & $\mathrm{TN}(\mathrm{mg} / \mathrm{L})$ & -0.54 & 0.53 \\
& $\mathrm{NH}_{4}(\mathrm{mg} / \mathrm{L})$ & 0.33 & -0.59 \\
& $\mathrm{NO}_{3}(\mathrm{mg} / \mathrm{L})$ & 0.58 & -0.50 \\
\hline Actinobacteria & $\mathrm{TP}(\mathrm{mg} / \mathrm{L})$ & 0.66 & -0.65 \\
\hline Firmicutes & $\mathrm{TP}(\mathrm{mg} / \mathrm{L})$ & -0.21 & 0.10 \\
\hline Planctomycetes & $\mathrm{pH}$ & 0.86 & 0.39 \\
\hline Thermotogae & $\mathrm{WT}$ & 0.72 & 0.40 \\
\hline
\end{tabular}
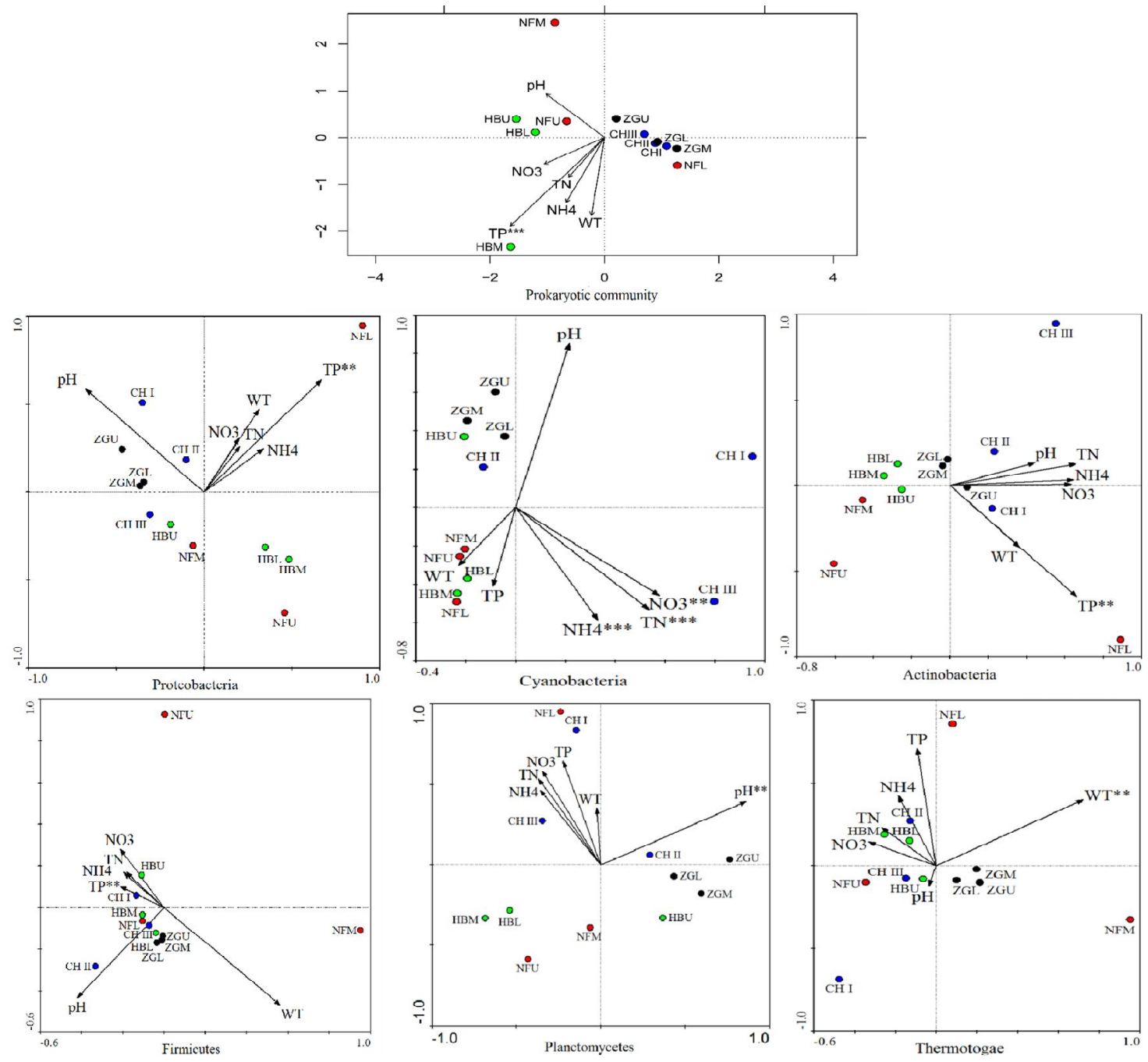

Figure 3. Redundancy analysis (RDA) biplots of prokaryotic communities and 8 prokaryotic phyla in correlation with environmental variables. Abbreviations are the same as those in Table 1. ***Significant at $p<0.01 ; * *$ Significant at $p<0.05$ 


\section{Discussion}

\section{Prokaryotic community structure characteristics}

High-throughput sequencing has been widely used to study differences in prokaryotic communities (Dai et al., 2016; Jordaan and Bezuidenhout, 2016; Liu et al., 2015; Yang et al., 2016). Bao (2008) analyzed the CCBs of Chaohu Lake, using a combination of PCR amplification of $16 \mathrm{~S}$ rRNA genes and DGGE, and reported that Chaohu Lake had 4 dominant phyla. In the present study, Chaohu Lake prokaryotes were distributed among 13 dominant phyla. Compared with the PCR-DGGE approach, high-throughput sequencing could identify more prokaryotic species, and is thus more suitable for describing prokaryotic community diversity in aquatic systems.

Proteobacteria, Cyanobacteria, Bacteroidetes, Actinobacteria, and Verrucomicrobia are the main prokaryotic phyla in freshwater ecosystems (Ren, 2013). Similarly, our results found Proteobacteria, Cyanobacteria, Bacteroidetes, and Actinobacteria to be the most common prokaryotic phyla of Chaohu Lake and its tributaries-results that are in agreement with those from previous studies (Wu et al., 2007; Dai et al., 2016; Jordaan and Bezuidenhout, 2016). Proteobacteria was the largest prokaryotic phylum of Chaohu Lake and its adjoining rivers, and exhibited high abundances in all samples, especially in Nanfei River, which showed the highest nutrient concentration. Proteobacteria abundance is known to be higher when environmental conditions are poor (Sun et al., 2016) and hence the present study suggests that the water quality of Chaohu Lake and its three tributaries is deteriorating.

Among the Proteoprokaryotic communities, Alphaproteobacteria, Betaproteobacteria, and Gammaproteobacteria dominated-with Betaproteobacteria exhibiting the highest abundance of the three. Betaproteobacteria represent one of the key components of freshwater bacterioplankton and play an important role in freshwater ecosystems (Crump and Hobbie, 2005; Newton et al., 2006; Salcher et al., 2013). Limnohabitans and Hydrogenophaga were the dominant genera of Betaproteobacteria. Limnohabitans belongs to BetI lineage of Betaproteobacteria, is the most abundant type of prokaryotes, and is widely distributed throughout of the world (Ren, 2013). Furthermore, Limnohabitans plays an important role in phosphorus cycling in eutrophic water and wastewater, and its distribution includes urban rivers (Jordaan and Bezuidenhout, 2016). The PhoD gene of the genus Limnohabitans codes the APase, which promotes the process of eutrophication by increasing the bioavailability of phosphorus (Zhao et al., 2015). Gammaproteobacteria and Alphaproteobacteria, the second and third classes of Proteobacteria, respectively, are found in a variety of aquatic habitats (Crump and Hobbie, 2005; Newton et al., 2011; Liu et al., 2012), but are generally more dominant in marine prokaryotic communities (Biers et al., 2009).

Hangbu River exhibited the highest abundance of Bacteroidetes, the second most abundant phylum. Within the Bacteroidetes, Flavobacterium was the most frequently detected genus-results that are consistent with bacterioplankton communities of Taihu Lake (Wu et al., 2007). Due to its algicidal activity and antagonism toward other bacteria, Flavobacterium has been associated with harmful algal blooms (Roth et al., 2008). Furthermore, it is often found in high abundance in eutrophic and hypertrophic urban rivers-usually correlating with high nutrient levels (Drury et al., 2013; Jordaan and Bezuidenhout, 2016).

Chaohu Lake and Zhegao River showed the highest abundances of Cyanobacteria, the third most dominant phylum. Cyanobacteria are the oldest known oxygen-producing 
organisms on Earth, and are widely distributed in freshwater ecosystems. Furthermore, these organisms are capable of adapting to environmental stresses, such as elevated nutrients (Paerl, 2008) and high temperatures (Briand et al., 2004). In shallow, nonstratified, lakes it has been suggested that the potential for cyanobacteria to dominate is greatest when the water temperature is high and the nutrient load is elevated (Elliot et al., 2006). In the present study, Cyanobacteria comprised four dominant genera: Dolichospermum, Prochlorococcus, and two unclassified genera. It should be noted that Dolichospermum is the most common genus in Cyanobacterial blooms (Liu et al., 2015).

Actinobacteria, the fourth dominant phylum in our study, are globally distributed and are often the most abundant phylum in a variety of freshwater ecosystems (Crump and Hobbie, 2005; Wilhelm et al., 2014). In Lake Taihu, a shallow hypertrophic freshwater lake in China, Actinobacteria are considered to be the most significant lineage (Wu et al., 2007).

Less abundant phylotypes were grouped with the Firmicutes, Verrucomicrobia, Chloroflexi, Planctomycetes, Acidobacteria, Chlorobi, Thermotogae, Crenarchaeota, Euryarchaeota, and unaffiliated. Firmicutes make up a small fraction of the prokaryotic community in some freshwater systems (Eiler and Bertilsson, 2004; Lymperopoulou et al., 2012), and predominantly consist of Gram-positive, rod-shaped, bacteria in the class Bacilli. Most of the species in this class exhibit strong environmental adaptability and can form stress resistant spores. Thus Bacilli play an important biological role in a variety of ecological systems, such as the treatment of industrial wastewater (Song et al., 2015). In this study, the highest abundance of Bacilli was found in the Nanfei River, in which the highest nutrient concentration was recorded. Verrucomicrobia exhibited a low abundance (1-4\%) in all of our sample collections. This phylum is widely distributed in rivers and lakes, and its low abundance may be associated with surplus nutrients (Haukka et al., 2006).

There are contradictory reports of Bacteroidetes abundance in the literature. For example, Bacteroidetes have been detected in high frequencies in lakes associated with Cyanobacterial blooms (Eiler and Bertilsson, 2004; Newton et al., 2011). However, this phylum has characteristically been found in association with clear water or mesotrophic lakes (Van der Gucht et al., 2005; McLaughlin et al., 2015). We found that Bacteroidetes were more abundant at sample stations exhibiting relatively low nutrient concentrations. In contrast, Actinobacteria and Cyanobacteria were primarily distributed among stations with high nutrient levels.

The recorded dominance of phylotypes belonging to Bacteroidetes, Betaproteobacteria, Alphaproteobacteria, Cyanobacteria, and Actinobacteria may be the result of the strong eutrophication of Chaohu Lake and its three tributaries. For example, similar results have been recorded for other eutrophic water bodies, and this may indicate a decline in water quality in the near future (Eiler and Bertilsson, 2004; Van Der Gucht et al., 2005; de Figueiredo et al., 2007; Wiedner et al., 2007; Wu et al., 2007). Furthermore, the CPCs is altered with the production of some groups such as Cyanobacteria and Actinobacteria, whose abundances may threaten water quality (Van Der Gucht et al., 2005; de Figueiredo et al., 2007; Wiedner et al., 2007). In the present study, Actinobacteria, Bacteroidetes, Alphaproteobacteria, Cyanobacteria, and Betaproteobacteria were co-dominant in Chaohu Lake and its three tributariesenvironments that were characterized as eutrophic or hypereutrophic. 


\section{Environmental effects on prokaryotic community}

The nutrient content of waterbodies has a significant impact on the diversity of prokaryotes (Lindström, 2000; Yan et al., 2017). Our RDA analysis showed that the prokaryotic communities and 3 prokaryotic phyla-including Proteobacteria, Actinobacteria, and Firmicutes-were significantly associated with TP. Furthermore, Cyanobacteria showed a significant correlation with $\mathrm{TN}, \mathrm{NH}_{4}$, and $\mathrm{NO}_{3}$. These findings are consistent with those of previous studies (Liu et al., 2011; Zhang et al., 2012; Niu et al., 2015; Wang et al., 2015). Of the measured explanatory variables, phosphorus and nitrogen were shown to have the greatest effect on CPCs, and this result may hold the greatest significance for the management of nature reserves. Specifically, understanding how phosphorus and nitrogen affect CPCs could aid in the search for new strategies to combat the degradation of freshwater ecosystems due to anthropogenic pollution.

Previous research has shown prokaryotic diversity to increase with elevated nutrient levels (Feng et al., 2007; Pang et al., 2014). However, other studies have found prokaryotic community diversity to significant decrease with the increase of freshwater nutrients (Van der Gucht et al., 2005; McLaughlin et al., 2015). Compared with the Zhegao and Hangbu Rivers, Nanfei River and Chaohu Lake had higher nutrient levels, and lower prokaryotic diversity. Our results have shown a link between higher waterbody nutrients and lower prokaryotic diversity. Microorganisms that can use and/or survive in the presence of pollutants often dominate contaminated ecosystems. As the result, the diversity of microbial communities is less than that of noncontaminated systems (McLaughlin et al., 2015). Van der Gucht et al. (2005) indicated that, as a result of the disappearance of submerged macrophyte stands, this transition is associated with a loss of structural diversity and, consequently, a decrease in biodiversity at higher trophic levels.

Our RDA results indicate that $\mathrm{pH}$ is correlated with Planctomycetes, this result is consistent with those of Ren et al. (2015), who observed that $\mathrm{pH}$ was the strongest determinant of both overall bacterioplankton community composition and the composition of abundant bacterioplankton groups. Previous literature has shown that water temperature can affect freshwater bacterioplankton communities (Adams et al., 2010; de Figueiredo et al., 2010; Zhang et al., 2012; Jordaan and Bezuidenhout, 2016). We observed that water temperature (WT) is generally associated with Thermotogae. Hence, this study suggests that $\mathrm{pH}$ and water temperature may play crucial roles in shaping bacterioplankton community structure in freshwater lakes and rivers.

\section{Conclusions}

In this study, CPCs were characterized using high-throughput sequencing and the relationship between CPCs and environment factors was explored in Chaohu Lake and its three urban tributaries. Our results indicated that elevated nutrients, $\mathrm{pH}$, and water temperature may be the primary environmental controllers of the variation in CPCs in Chaohu Lake and its three tributaries. Furthermore, we found that the variation of CPCs was influenced by anthropogenic perturbations-eutrophication processes that are continuing to increase within the Chaohu Lake watershed. Ultimately, these results add to our growing understanding of the ecology of CPCs in Chaohu Lake and its tributaries, and may offer crucial ecological background data for the development of management plans for this important urban freshwater system. 
Acknowledgements. This research was supported by the CRSRI Open Research Program (CKWV2015238/KY) and the Visiting Research Foundation at Home and Abroad for the Outstanding Young Backbone Talents of Colleges and Universities of Anhui Province (No. gxfx2017082).

\section{REFERENCES}

[1] Adams, H. E., Crump, B. C., Kling, G. W. (2010): Temperature controls on aquatic bacterial production and community dynamics in arctic lakes and streams. Environmental Microbiology 12: 1319-1333.

[2] Bao, S. M. (2008): Horizontal and Seasonal Dynamics of the Bacterioplankton Community Composition in the Large Shallow Chaohu Lake, China. - Anhui Agricultural University, Hefei.

[3] Bashenkhaeva, M. V., Zakharova, Y. R., Petrova, D. P., Khanaev, I. V., Galachyants, Y. P., Likhoshway, Y. V. (2015): Sub-ice microalgal and bacterial communities in freshwater Lake Baikal Russia. - Microbial Ecology 70: 751-765.

[4] Biers, E. J., Sun, S. L., Howard, E. C. (2009): Prokaryotic genomes and diversity in surface ocean waters: interrogating the global ocean sampling metagenome. - Applied and Environmental Microbiology 75(7): 2221-2229.

[5] Briand, J. F., Leboulanger, C., Humbert, J. F. (2004): Cylindrospermopsis raciborskii (cyanobacteria) invasion at mid-latitudes: selection wide physiological tolerance or global warming. - Journal of Phycology 40: 231-238.

[6] Caporaso, J. G., Kuczynski, J., Stombaugh, J., Bittinger, K., Bushman, F. D., Costello, E. K., Fierer, N., Pena, A. G., Goodrich, J. K., Gordon, J. I., Huttley, G. A., Kelley, S. T., Knights, D., Koenig, J. E., Ley, R. E., Lozupone, C. A., McDonald, D., Muegge, B. D., Pirrung, M., Reeder, J., Sevinsky, J. R., Turnbaugh, P. J., Walters, W. A., Widmann, J., Yatsunenko, T., Zaneveld, J., Knight, R. (2010): QIIME allows analysis of highthroughput community sequencing data. - Nature Methods 7(5): 335-336.

[7] Crump, B. C., Hobbie, J. E. (2005): Synchrony and seasonality in bacterioplankton communities of two temperate rivers. - Limnology and Oceanography 50(6): 1718-1729.

[8] Dai, Y., Yang, Y. Y., Wu, Z., Feng, Q. Y., Xie, S. G., Liu, Y. (2016): Spatiotemporal variation of planktonic and sediment bacterial assemblages in two plateau freshwater lakes at different trophic status. - Applied Microbiology and Biotechnology 100: 41614175 .

[9] de Figueiredo, D. R., Pereira, M. J., Moura, A., Silva, L., Barrios, S., Fonseca, F., Henriques, I., Correia, A. (2007): Bacterial community composition over a dry winter in meso- and eutrophic Portuguese water bodies. - FEMS Microbiology Ecology 59: 638650 .

[10] de Figueiredo, D. R., Pereira, M. J., Correia, A. (2010): Seasonal modulation of bacterioplankton community at a temperate eutrophic shallow lake. - World Journal of Microbiology \& Biotechnology 26: 1067-1077.

[11] Drury, B., Rosi-Marshall, E., Kelly, J. J. (2013): Wastewater treatment effluent reduces the abundance and diversity of benthic bacterial communities in urban and suburban rivers. - Applied and Environmental Microbiology 79(6): 1897-1905.

[12] Edgar, R. C., Haas, B. J., Clemente, J. C., Quince, C., Knight, R. (2011): UCHIME improves sensitivity and speed of chimera detection. - Bioinformatics 27: 2194-2200.

[13] Eiler, A., Bertilsson, S. (2004): Composition of freshwater bacterial communities associated with cyanobacterial blooms in four Swedish lakes. - Environmental Microbiology 6: 1228-1243.

[14] Elliot, J. A., Jones, I. D., Thackeray, S. J. (2006): Testing the sensitivity of phytoplankton communities to changes in water temperature on nutrient load in a temperate lake. Hydrobiologia 559: 401-411. 
[15] Feng, S., Qin, B. Q., Gao, G. (2007): Response of bacterial communities to eutrophic water in Lake Taihu. - Acta Scientiae Circumstantiae 27(11): 1823-1829.

[16] Haukka, K., Kolmonen, E., Hyder, R., Hietala, J., Vakkilainen, K., Kairesalo, T., Haario, H., Sivonen, K. (2006): Effect of nutrient loading on bacterioplankton community composition in lake mesocosms. - Microbial Ecology 51(2): 137-146.

[17] Huang, X. F. (2000): Survey, Observation and Analysis of Lake Ecology. - Standard Press of China, Beijing.

[18] Jordaan, K., Bezuidenhout, C. C. (2016): Bacterial community composition of an urban river in the North West Province South Africa in relation to physico-chemical water quality. - Environmental Science and Pollution Research 23: 5868-5880.

[19] Lindström, E. S. (2000): Bacterioplankton community composition in five lakes differing in trophic status and humic content. - Microbial Ecology 40: 104-113.

[20] Liu, L. M., Yang, J., Zhang, Y. Y. (2011): Genetic diversity patterns of microbial communities in a subtropical riverine ecosystem (Jiulong River southeast China). Hydrobiologia 678: 113-125.

[21] Liu, L. M., Yang, J., Yu, Z., Wilkinson, D. M.. (2015): The biogeography of abundant and rare bacterioplankton in the lakes and reservoirs of China. - ISME Journal 9: 20682077.

[22] Liu, Y., Gao, H. Y., Li, X. Y., Li, R. H. (2015): Identification and determination of mycosporine-like amino acids (MAAs) in dolichospermum flos-aquae. - Acta Hydrobiologica Sinica 39(3): 549-553.

[23] Liu, Z. H., Huang, S. B., Sun, G. P., Xu, Z. C., Xu, M. Y. (2012): Phylogenetic diversity composition and distribution of bacterioplankton community in the Dongjiang River China. - FEMS Microbiology Ecology 80(1): 30-44.

[24] Lin, X. J., McKinley, J., Resch, C. T., Kaluzny, R., Lauber, C. L., Fredrickson, J., Knight, R., Konopka, A. (2012): Spatial and temporal dynamics of the microbial community in the Hanford unconfined aquifer. - ISME Journal 6: 1665-1676.

[25] Lymperopoulou, D. S., Kormas, K. Ar., Karagouni, A. D. (2012): Variability of prokaryotic community structure in a drinking water Reservoir (Marathonas Greece). Microbes and Environments 27(1): 1-8.

[26] Magoc, T., Salzberg, S. L. (2011): FLASH: Fast length adjustment of short reads to improve genome assemblies. - Bioinformatics 27: 2957-2963.

[27] Martinuzzi, S., Januchowski-Hartley, S. R., Pracheil, B. M., McIntyre, P. B., Plantinga, A. J., Lewis, D. J., Lewis, D. J., Radeloff, V. C. (2014): Threats and opportunities for freshwater conservation under future land use change scenarios in the United States. Global Change Biology 20(1): 113-24.

[28] McLaughlin, R. W., Wang, S. B., Zhou, W., Cheng, G. J., Deng, K. J. (2015): A comparison of the bacterial diversity of two shallow freshwater lakes in China. Proceedings of the National Academy of Sciences, India Section B: Biological Sciences 85(1): 137-146.

[29] Newton, R. J., Kent, A. D., Triplett, E. W., Mcmahon, K. D. (2006): Microbial community dynamics in a humic lake: differential persistence of common freshwater phylotypes. - Environmental Microbiology 8(6): 956-970.

[30] Newton, R. J., Jones, S. E., Eiler, A., Mcmahon, K. D., Bertilsson, S. (2011): A Guide to the natural history of freshwater lake bacteria. - Microbiology and Molecular Biology Reviews 75(1): 14-49.

[31] Ni, J. J., Li, X. J., He, Z. L., Xu, M. Y. (2017): A novel method to determine the minimum number of sequences required for reliable microbial community analysis. Journal of Microbiological Methods 139: 196-201.

[32] Niu,Y., Yu, H., Jiang, X. (2015): Within-lake heterogeneity of environmental factors structuring bacterial community composition in Lake Dongting China. - World Journal of Microbiology \& Biotechnology 31: 1683-168. 
[33] Oksanen, J., Blanchet, F. G., Kindt, R., Legendre, P., Minchin, P. R., OHara, R. B., Simpson, G. L., Solymos, P., Stevens, M. H. H., Wagner, H. (2015): Vegan: Community Ecology Package R Package Version 22-1. - http://CRANR-projectorg/package=vegan.

[34] Organisation for Economic and Co-operation and Development (OECD) (1982): Eutrophication of Waters: Monitoring Assessment and Control. - OECD, Paris.

[35] Paerl, H. W. (2008): Nutrient and other environmental controls of harmful cyanobacterial blooms along the freshwater-marine continuum. - Advances in Experimental Medicine and Biology 619: 216-241.

[36] Pang, X. H., Lü, L. Y., Niu, Y., Shen, H., Yuan, X. G., Chen, W. J., Chen, J., Xie, P. (2014): The spatial pattern of bacterioplankton communities composition in summer in Lake Taihu. - Acta Hydrobiologica Sinica 38(2): 335-341.

[37] Ren, L. J., He, D., Xing, P., Wang, Y. J., Wu, Q. L. (2013): Bacterial diversity and ecological function in lake water bodies. - Biodiversity Science 21(4): 421-432.

[38] Ren, L. J., Jeppesen, E., He, D., Wang, J. J., Liboriussen, L., Xing, P., Wu, Q. L. (2015): $\mathrm{pH}$ influences the importance of niche-related and neutral processes in lacustrine bacterioplankton assembly. - Applied and Environmental Microbiology 81(9): 31043114.

[39] Roth, P. A., Mikulski, C. M., Doucette, G. J. (2008): The influence of microbial interactions on the susceptibility of Karenia spp to algicidal bacteria. - Aquatic Microbial Ecology 50: 251-259.

[40] Salcher, M. M., Posch, T., Pernthaler, J. (2013): In situ substrate preferences of abundant bacterioplankton populations in a prealpine freshwater lake. - ISME Journal 7(5): 896907.

[41] Song, Z. Q., Wang, L., Liu, X. H., Liang, F. (2015): Diversities of Firmicutes in four hot springs in Yunnan and Tibet. - Biotechnology 25(5): 481-486.

[42] Sun, Y. J., Wang, T. Y., Peng, X. W., Wang, P., Lu, Y. L. (2016): Bacterial community compositions in sediment polluted by perfluoroalkyl acids (PFAAs) using Illumina highthroughput sequencing. - Environmental Science and Pollution Research 23: 1055610565.

[43] Van der Gucht, K., Vandekerckhove, T., Vloemans, N., Cousin, S., Muylaert, K., Sabbe, K., Gillis, M., Declerk, S., De Meester, L., Vyverman, W. (2005): Characterization of bacterial communities in four freshwater lakes differing in nutrient load and food web structure. - FEMS Microbiology Ecology 53: 205-220.

[44] Wang, Q., Garrity, G. M., Tiedje, J. M., Cole, J. R. (2007): Naïve Bayesian classifier for rapid assignment of rRNA sequences into the new bacterial taxonomy. - Applied and Environmental Microbiology 73: 5261-5267.

[45] Wang, S., Jiang, X., Jin, X. (2011): Classification and pollution characteristic analysis for inflow rivers of lake Chaohu. - Environmental Science 32: 2834-2839.

[46] Wang, S. M., Dou, H. S. (1998): Biography of Lakes in China. - Science Press, Beijing.

[47] Wang, Y. M., Yang, J., Liu, L. M., Yu, Z. (2015): Quantifying the effects of geographical and environmental factors on distribution of stream bacterioplankton within nature reserves of Fujian China. - Environmental Science and Pollution Research 22: 1101011021.

[48] Wei, C. L., Bao, S. M., Zhu, X. Y., Huang, X. M. (2008): Spatio-temporal variations of the bacterioplankton community composition in Chaohu Lake China. - Progress in Natural Science 18: 1115-1122.

[49] Wiedner, C., Rücker, J., Bruggemann, R., Nixdorf, B. (2007): Climate change affects timing and size of populations of an invasive cyanobacterium in temperate regions. Oecologia 152: 473-484.

[50] Wilhelm, S. W., Le Cleir, G. R., Bullerjahn, G. S., McKay, R. M., Saxton, M. A., Twiss, M. R., Bourbonniere, R. A. (2014): Seasonal changes in microbial community structure and activity imply winter production is linked to summer hypoxia in a large lake. - FEMS Microbiology Ecology 87(2): 475-485. 
[51] Wu. L., Sun, Q. Y., Ni, J. J. (2017): Not all of the rare operational taxonomic units (OTUs) play the same role in maintaining community stability. - Applied Ecology and Environmental Research 15(1): 105-112.

[52] Wu, X., Xi, W., Ye, W., Yang, H. (2007): Bacterial community composition of a shallow hypertrophic freshwater lake in China revealed by $16 \mathrm{~S}$ rRNA gene sequences. - FEMS Microbiology Ecology 61: 85-96.

[53] Xiang, J. G., He, T. Y., Wang, P. P., Xie, M., Xiang, J., Ni, J. J. (2018): Opprtunistic pathogens are abundant in the gut of cultured giant spiny frog (Paa spinosa). Aquaculture Research 49: 2033-2041.

[54] Yan, Q. Y., Stegen, J. C., Yu, Y. H., Deng, Y., Li, X. H., Wu, S., Dai, L. L., Zhang, X., Li, J. J., Wang, C., Ni, J. J., Li, X. M., Hu, H. J., Xiao, F. S., Feng, W. S., Ning, D. L., He, Z. L., Van Nostrand, J. D., Wu, L. Y., Zhou, J. Z. (2017): Nearly a decade-long repeatable seasonal diversity patterns of bacterioplankton communities in the eutriphic Lake Donghu (Wuhan China). - Molecular Ecology 26: 3839-3850.

[55] Yang, J., Jiang, H. C., Wu, G., Liu, W., Zhang, G. J. (2016): Distinct factors shape aquatic and sedimentary microbial community structures in the lakes of western China.Frontiers in Microbiology 08: 1-8.

[56] Zan, F. Y., Huo, S. L., Xi, B. D., Li, Q. Q., Liao, H. Q., Zhang, J. T. (2011): Phosphorus distribution in these dements of a shallow eutrophic lake, Lake Chaohu, China. Environmental Earth Sciences 62: 1643-1653.

[57] Zhao, D. D., Luo, J. F., Huang, X. Y., Lin, W. T. (2015): Diversity of bacterial APase phoD gene in the Pearl River water. - Acta Scientiae Circumstantiae 35(3): 722-728.

[58] Zhang, M. L., Yu, N., Chen, L. Q., Jiang, C. H., Tao, Y. J., Zhang, T., Chen, J., Xue, D. (2012): Structure and seasonal dynamics of bacterial communities in three urban rivers in China. - Aquatic Sciences 74: 113-12. 